\title{
Fabrication of a Silicide Thermoelectric Module Employing Fractional Factorial Design Principles
}

\author{
Joachim S. Graff ${ }^{1} \cdot$ Raphael Schuler $^{2} \cdot$ Xin Song $^{3} \cdot$ Gustavo Castillo-Hernandez ${ }^{4} \cdot$ Gunstein Skomedal $^{5}$. \\ Erik Enebakk $^{5}$. Daniel Nilsen Wright ${ }^{6}\left({ }^{\circ}\right.$. Marit Stange ${ }^{1}$. Johannes de Boor ${ }^{4}$. Ole Martin Løvvik ${ }^{1,3}$. \\ Matthias Schrade ${ }^{1}$ (i)
}

Received: 9 December 2020 / Accepted: 19 March 2021 / Published online: 23 April 2021

(c) The Author(s) 2021

\begin{abstract}
Thermoelectric modules can be used in waste heat harvesting, sensing, and cooling applications. Here, we report on the fabrication and performance of a four-leg module based on abundant silicide materials. While previously optimized $\mathrm{Mg}_{2} \mathrm{Si}_{0.3} \mathrm{Sn}_{0.675} \mathrm{Bi}_{0.025}$ is used as the $n$-type leg, we employ a fractional factorial design based on the Taguchi methods mapping out a four-dimensional parameter space among $\mathrm{Mn}_{\mathrm{x}-\varepsilon} \mathrm{Mo}_{\varepsilon} \mathrm{Si}_{1.75-\delta} \mathrm{Ge}_{\delta}$ higher manganese silicide compositions for the $p$-type material. The module is assembled using a scalable fabrication process, using a $\mathrm{Cu}$ metallization layer and a $\mathrm{Pb}$-based soldering paste. The maximum power output density of $53 \mu \mathrm{W} \mathrm{cm}{ }^{-2}$ is achieved at a hot-side temperature of $250{ }^{\circ} \mathrm{C}$ and a temperature difference of $100{ }^{\circ} \mathrm{C}$. This low thermoelectric output is related to the high contact resistance between the thermoelectric materials and the metallic contacts, underlining the importance of improved metallization schemes for thermoelectric module assembly.
\end{abstract}

Keywords Thermoelectric materials $\cdot$ Taguchi method $\cdot$ silicides $\cdot$ thermoelectric generator

\section{Introduction}

Thermoelectric (TE) materials, which can convert thermal into electrical energy exploiting the Seebeck effect, have a huge potential in various waste heat recovery and active cooling applications, where scalability, longevity and noiselessness compensate for the rather low conversion efficiency. ${ }^{1,2}$

Matthias Schrade

matthias.schrade@sintef.no

1 Department of Sustainable Energy Technology, SINTEF Industry, 0373 Oslo, Norway

2 Department of Chemistry, Centre for Materials Science and Nanotechnology, University of Oslo, 0318 Oslo, Norway

3 Department of Physics, Centre for Materials Science and Nanotechnology, University of Oslo, 0316 Oslo, Norway

4 Institute of Materials Research, German Aerospace Center, 51170 Cologne, Germany

5 Elkem ASA, 4675 Kristiansand, Norway

6 Department of Microsystems and Nanotechnology, SINTEF DIGITAL, 0373 Oslo, Norway
However, most commercial thermoelectric devices are based on scarce, expensive or toxic elements, like $\mathrm{Te}$ and $\mathrm{Pb}$, and much research effort is devoted to finding new materials based on environmentally friendly and benign elements, which combine excellent TE performance with good temperature stability. The potential of different materials for TE applications is usually compared by the thermoelectric figure of merit, $z T=\alpha^{2} \sigma / \kappa \times T$, where $\alpha$ is the Seebeck coefficient, $\sigma$ the electrical conductivity and $\kappa$ the thermal conductivity.

Several classes of materials are identified as promising thermoelectrics in the temperature range from $300{ }^{\circ} \mathrm{C}$ to $800{ }^{\circ} \mathrm{C}$, for example, half-Heusler compounds, ${ }^{3,4}$ skutterudites, ${ }^{5}$ Zintl phases ${ }^{6}$ and some members of the silicide family. ${ }^{7}$ For example, acceptor-doped $\mathrm{Mg}_{2} \mathrm{Si}$-based compounds (MGS) (usually isoelectrically alloyed with $\mathrm{Sn}$ on the Si sublattice to reduce the thermal conductivity $\kappa$ ) show $z T$ values of up to 1.5 at $523{ }^{\circ} \mathrm{C},{ }^{8-10}$ with much lower values for the corresponding $p$-type compositions, due to limited dopant efficiency and unfavorable properties of the valence bands compared to the conduction bands. ${ }^{11-14}$ Good $p$-type TE properties are also found among the higher manganese silicides (HMS), with an approximate stoichiometry of $\mathrm{MnSi}_{\gamma}$ 
$(\gamma \approx 1.75)$, reaching $z T$ of 0.8 at $600{ }^{\circ} \mathrm{C},{ }^{15}$ but the mismatch in the thermal expansion coefficient (CTE) between $\mathrm{Mg}_{2} \mathrm{Si}$ of $17 \times 10^{-6} \mathrm{~K}^{-1}$ and HMS of $13 \times 10^{-6} \mathrm{~K}^{-1}$ limits reliable integration into TE modules intended for long-term operation under large temperature gradients. ${ }^{16}$ As a consequence, only few reports exist in the literature on the fabrication of all-silicide TE modules. ${ }^{17-21}$

Progress in TE material discovery and optimization is notoriously slow and incremental due to the large number of independent parameters with often detrimental effect on the various functional and mechanical properties. For example, these parameters include the overall chemical composition of the investigated samples, but also include fabrication parameters, like synthesis route and sintering temperature. A complete, systematic mapping of this multidimensional parameter space is associated with immense investments of resources and time and therefore practically illusional.

As an alternative, we here employ a fractional factorial experimental design concept based on the Taguchi methods. ${ }^{22,23}$ So far, within thermoelectric research, this approach has been used mostly for geometric optimization of module dimensions. ${ }^{24-28}$ Few studies use a fractional experimental design to find robust processing parameters, ${ }^{29,30}$ but screening several independent compositional parameters, as attempted in this study, has not yet been reported in the literature. The main idea behind the Taguchi methods is to span a large parameter space with a minimum of orthogonal base vectors, each representing a different combination of the individual parameters. Instead of systematically testing all possible combinations of the investigated parameters, major trends can be deduced based on a dramatically reduced set of samples. A requirement of this approach is the absence of mutual correlation between the parameters, and good control of additional properties, which could also influence the outcome but are not included in the set of tested parameters. For our experiments, we chose the L9 orthogonal array, as it illustrates the capabilities of Taguchi's approach: as shown in Table I, four different parameters can be tested at a time, each at three different predefined levels. If all combinations of parameters and levels were to be tested, this would require $3^{4}=81$ individual runs; with the experimental design plan of the Taguchi methods, a robust result within the same parameter space is obtained within only nine runs.

Here, we use this Taguchi method at two different stages: once to find suitable sintering parameters for achieving dense and mechanically robust samples, and subsequently to screen the composition for dually doped $\mathrm{Mn}_{\mathrm{x}-\varepsilon} \mathrm{Mo}_{\varepsilon} \mathrm{Si}_{1.75-\delta} \mathrm{Ge}_{\delta} \mathrm{HMS}$ compounds. For the statistical analysis, we make use of analysis of variance (ANOVA) tables, as described in various reports, e.g. Ref. 24. Finally, we synthesize larger quantities of the chosen HMS composition $\mathrm{Mn}_{1.01} \mathrm{Si}_{1.745} \mathrm{Ge}_{0.005}$ and previously optimized $\mathrm{Mg}_{2} \mathrm{Si}$ to test their compatibility within a thermoelectric module. The goal of the current study is to
Table I The L9 array used for the fractional experimental design in this study

\begin{tabular}{lllll}
\hline Run & \multicolumn{2}{l}{ Parameter } & & \\
\cline { 2 - 5 } & A & B & C & D \\
\hline 1 & Low & Medium & Medium & Low \\
2 & Low & High & High & Medium \\
3 & Low & Low & Low & High \\
4 & Medium & Low & High & Low \\
5 & High & High & Low & Low \\
6 & Medium & Medium & Low & Medium \\
7 & High & Low & Medium & Medium \\
8 & Medium & High & Medium & High \\
9 & High & Medium & High & High \\
\hline
\end{tabular}

demonstrate the potential fractional factorial design principles in thermoelectric research. In addition, we develop scalable processes for the powder synthesis and assembly of TE modules.

\section{Experimental}

\section{Powder Synthesis}

Batches of the specific nominal compositions containing $\mathrm{Mn}, \mathrm{Mo}, \mathrm{Si}$ and Ge were prepared from elemental raw materials, employing easily scalable synthesis methods, adapted from our industrial partner ELKEM, one of the world's largest producers of silicon and related materials. Each batch was loaded in a top-covered induction furnace and heated under inert atmosphere to minimize surface oxidation. The materials were heated fast up to $1400{ }^{\circ} \mathrm{C}$, held for $10 \mathrm{~min}$ and then further heated to a temperature above the melting point of silicon. This temperature was then held for around $30 \mathrm{~min}$ to ensure that all material was melted and well mixed. The melt was poured into a graphite mold with casting thickness of approximately $5 \mathrm{~mm}$, ensuring a high cooling rate. The cast material was crushed, milled and sieved to obtain the final particle size distribution.

\section{Spark Plasma Sintering}

Powders were loaded into graphite dies and compacted into bulk pellets using a spark plasma sintering (SPS) instrument (Dr. Sinter 825). In the first step, we used the fractional factorial experimental design to find suitable sintering parameters.

During the screening of parameters and composition, we used dies with 20-mm diameter and fabricated cylindrical pellets with a thickness of ca. $2 \mathrm{~mm}$. For the production of pellets for the thermoelectric module, the die diameter 
and thickness were increased to $36 \mathrm{~mm}$ and $5 \mathrm{~mm}$, respectively. To minimize oxidation of the powder, the samples were loaded in the dies inside a glove bag which had been flushed with inert gas three times.

\section{Characterization Tools}

Microstructural and chemical analysis of polished surfaces of the fabricated samples was done by scanning electron microscopy (SEM) (FEI Nova NanoSEM 650) equipped with an energy-dispersive spectrometer (EDS) (Oxford Instruments Xmax 50).

The electrical resistivity and the Seebeck coefficient were measured under argon atmosphere using an apparatus described elsewhere. ${ }^{31}$ The thermal diffusivity was measured under nitrogen flow using the laser flash technique (Netzsch LFA 451). $\kappa$ was obtained from the thermal diffusivity $D$ by $\kappa=D \rho c_{\mathrm{p}}$ using the mass density $\rho$ and the specific heat capacity $c_{\mathrm{p}}$, as deducted by comparison of the laser flash output signal with a Pyroceram reference sample.

\section{Module Fabrication}

Fabricated disks were cut into rectangular legs using a diamond-plated cutting wheel. The height of the individual legs was adjusted to be within $10 \mu \mathrm{m}$ to ensure physical contact during the module assembly process. Contacting surfaces were polished before metallization. A thin copper layer

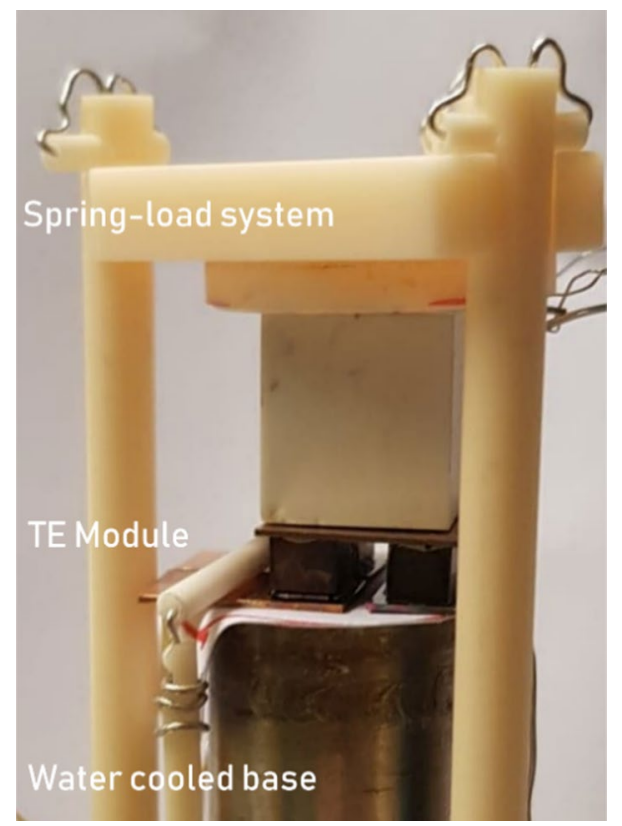

Fig. 1 A photograph of the setup used for module characterization, as described in the text.
Table II The parameters used to vary the sintering conditions. See Fig. 2 for a graphical definition of the four different parameters.

\begin{tabular}{|c|c|c|c|c|}
\hline \multirow[t]{2}{*}{ Level } & \multicolumn{4}{|l|}{ Parameters } \\
\hline & $\mathrm{A}: T_{\mathrm{Max}}\left[{ }^{\circ} \mathrm{C}\right]$ & $\begin{array}{l}\text { B: } t_{\text {hold }} \text { at } \\
T_{\text {Max }} \text { and } \\
p_{\text {Max }} \text { [min] }\end{array}$ & $\begin{array}{l}\text { C: Time } p_{\mathrm{Max}} \\
\rightarrow 30 \mathrm{MPa} \\
{[\mathrm{min}]}\end{array}$ & $\mathrm{D}: t_{\mathrm{Cool}}[\mathrm{min}]$ \\
\hline Low & 750 & 1 & 1 & 10 \\
\hline Medium & 800 & 4 & 4 & 20 \\
\hline High & 850 & 20 & 20 & 40 \\
\hline
\end{tabular}

was deposited on the top and bottom surface with radiofrequency (RF) magnetron sputtering using a Polyteknik Flextura 200 cluster instrument with a deposition power of $200 \mathrm{~W}$, a background Ar pressure of $3 \times 10^{-3}$ mbar and a deposition time of $10 \mathrm{~min}$.

For module assembly, we used a purpose-made placement jig. Bonding between the metallized TE legs and the $\mathrm{Cu}$ contact strips was made using soldering paste (Felder $\mathrm{S}-\mathrm{Pb} 93 \mathrm{Sn} 5 \mathrm{Ag} 2$ ) dispensed on the $\mathrm{Cu}$ strips, before manually placing the legs. $\mathrm{Cu}$ strips on the top side of the module were then attached using soldering paste. A Cu plate (ca. $200 \mathrm{~g}$ ) was placed on top of the module to ensure good and homogeneous physical contact between the different components during bonding. The preassembled module was placed on a programmable hot plate and bonded in air at a maximum temperature of $315^{\circ} \mathrm{C}$. The thermoelectric performance of the module was then characterized under an argon atmosphere by a purpose-made setup (Fig. 1), described in detail in Ref. 32. The module is placed on a water-cooled cylinder and hold in place by a spring-load system. Top- and bottom temperatures are monitored using two S-type thermocouples placed, respectively, on top and under the module. The setup is fit into a high temperature measurement cell (ProboStat, NorECs, Norway), flushed with Argon and placed in a vertical tube furnace, providing the overall base temperature.

\section{Results and Discussion}

\section{Sintering of Pellets}

In the first step, we used the L9 orthogonal matrix, Table I, to investigate the influence of different processing parameters on the obtained sample density after sintering. We chose four independent parameters to be varied, each with three different values, as specified in Table II and illustrated in Fig. 2. For example, the "Run 1" experiment used a $T_{\mathrm{Max}}$ of $750{ }^{\circ} \mathrm{C}$, a $t_{\mathrm{Hold}} @ p_{\mathrm{Max}}+T_{\mathrm{Max}}$ of $4 \mathrm{~min}, t_{\mathrm{pMax} \rightarrow 30 \mathrm{MPa}}$ of $4 \mathrm{~min}$ and $t_{\mathrm{Cool}}$ of $10 \mathrm{~min}$. The heating rate in all runs was kept constant at $100 \mathrm{~K} / \mathrm{min}$, as we have found in preliminary experiments that it did not have a significant 
influence on the obtained results. The maximum pressure of $90 \mathrm{MPa}$ was kept constant for all runs. An ingot powder with a nominal stoichiometry of $\mathrm{MnSi}_{1.75}$ was used for the sintering experiments.

Assuming a theoretical density of $5.15 \mathrm{~g} \mathrm{~cm}^{-3}$ for $\mathrm{MnSi}_{1.75},{ }^{33}$ the relative mass density of all produced pellets

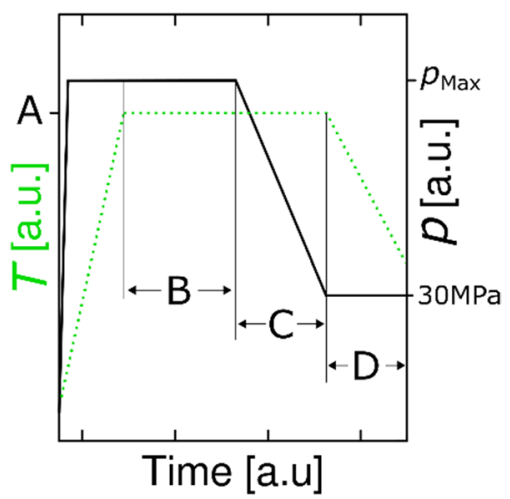

Fig. 2 Graphical representation of varied parameters, as given in Table II. is $>95 \%$, independent of the chosen parameters. As can be seen in Fig. 3 and Table III, the maximum temperature has the biggest effect on the obtained density, while parameter $\mathrm{B}, t_{\text {hold }}$ at $T_{\text {Max }}$ and $p_{\text {Max }}$, does not affect the result significantly. Note that the L9 orthogonal array does not contain any replicate measurements, so that it is not possible to differentiate between variance due to a change in parameters or random experimental variation (the degree of freedom for the "Error" line is 0). As several of the samples sintered at $850{ }^{\circ} \mathrm{C}$ broke into multiple pieces, we reduced $T_{\text {Max }}$ to $820^{\circ} \mathrm{C}$ in the subsequent experiments. As the other process parameters only have a minor influence on the obtained sample density, these parameters were set to $t_{\text {hold }} @ T_{\text {Max }}+p_{\text {Max }}$ $=2 \mathrm{~min}, t_{p \mathrm{Max}->30 \mathrm{MPa}}=10 \mathrm{~min}$ and $t_{\mathrm{Cool}}=20 \mathrm{~min}$, compromising the results from the Taguchi approach and a short synthesis time per sample.

\section{Varying Composition}

After finding suitable sintering parameters, we used the same fractional factorial design approach to vary the chemical composition of the samples with respect to their
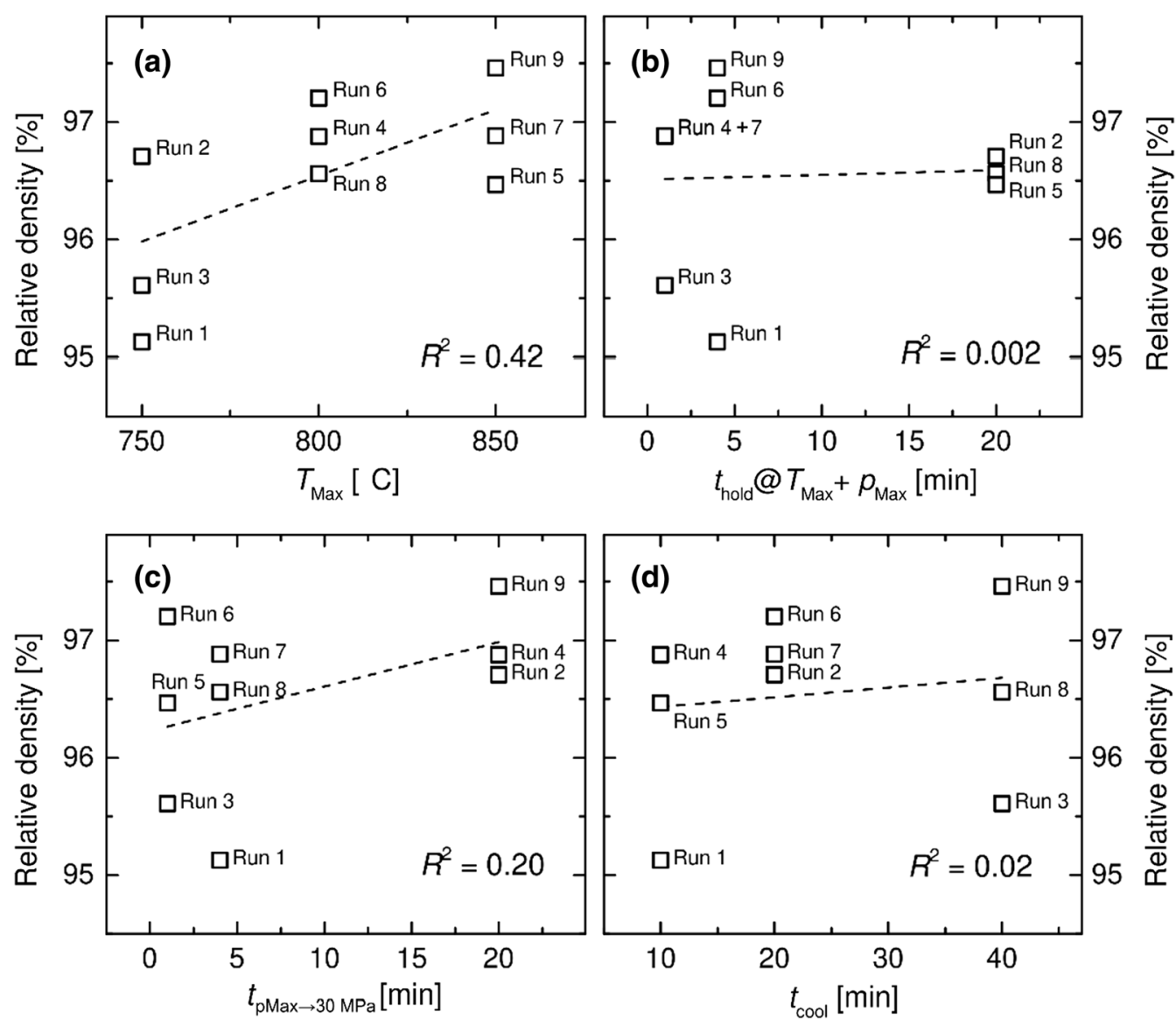

Fig. 3 Relative density of sintered samples as a function of the parameters described in Table II. (a) $T_{\text {Max }}$, (b) $t_{\text {hold }}$ at $T_{\text {Max }}$ and $p_{\text {Max }}$, (c) time $p_{\text {Max }}$ $\rightarrow 30 \mathrm{MPa}$ and (d) $t_{\mathrm{Cool}}$. 
Table III ANOVA table for a fractional experimental design to identify suitable sintering conditions.

\begin{tabular}{llll}
\hline Parameter & $\begin{array}{l}\text { Degrees of } \\
\text { freedom }\end{array}$ & $\begin{array}{l}\text { Sum of squares } \\
{\left[10^{-4}\right]}\end{array}$ & $\begin{array}{l}\text { Contri- } \\
\text { bution } \\
{[\%]}\end{array}$ \\
\hline$T_{\text {Max }}$ & 2 & 2.4 & 54.4 \\
$t_{\text {hold }}$ at $T_{\text {Max }}$ and $p_{\text {Max }}$ & 2 & 0.04 & 0.8 \\
Time $p_{\text {Max }} \rightarrow 30 \mathrm{MPa}$ & 2 & 1.1 & 24.6 \\
$t_{\text {Cool }}$ & 2 & 0.9 & 20.2 \\
Error & - & N/A & N/A \\
Total & 8 & 4.44 & 100 \\
\hline
\end{tabular}

Table IV The parameters used to optimize the composition of the $\mathrm{Mn}_{\mathrm{x}-\varepsilon} \mathrm{Mo}_{\varepsilon} \mathrm{Si}_{1.75-\delta} \mathrm{Ge}_{\delta} p$-type material

\begin{tabular}{lllll}
\hline Level & \multicolumn{4}{l}{ Parameters } \\
\cline { 2 - 5 } & A: $\delta$ & B: Powder processing & C: $x$ & D: $\varepsilon$ \\
\hline Low & 0 & Regular milling & 0.99 & 0 \\
Medium & 0.005 & Regular milling and sieving & 1.00 & 0.005 \\
High & 0.010 & Long milling without sieving & 1.01 & 0.020 \\
\hline
\end{tabular}
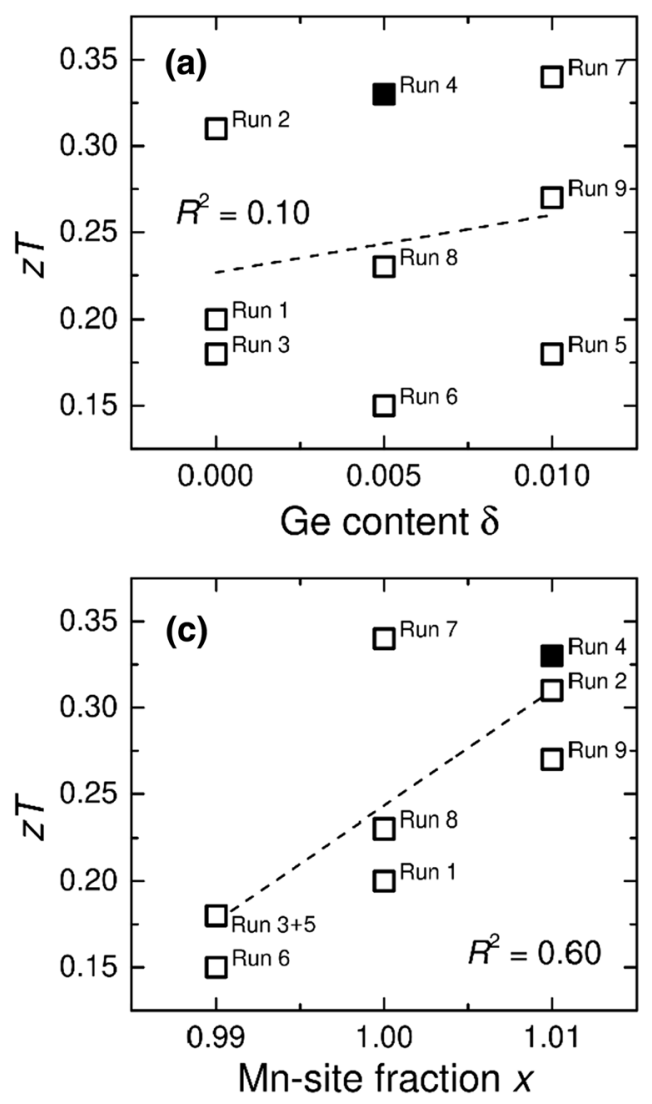

Fig.4 zT @ $400{ }^{\circ} \mathrm{C}$ of $\mathrm{Mn}_{\mathrm{x}-\varepsilon} \mathrm{Mo}_{\varepsilon} \mathrm{Si}_{1.75-\delta} \mathrm{Ge}_{\delta}$ as a function of different process parameters. (a) Ge doping on the Si site. (b) Milling/sieving of powder prior to sintering. (c) Mn site fraction $x$. (d) Mo doping on thermoelectric figure of merit $z T$. Mo and Ge doping have been demonstrated to increase the carrier concentration and therefore $z T,{ }^{34-36}$ while a variation of the $\mathrm{Mn}$ site fraction is expected to lead to changes in the crystal structure and phase compositions. As a fourth parameter, we varied the processing of the ingot powder. The overall stoichiometry of the ingot powders can be written as $\mathrm{Mn}_{\mathrm{x}-\varepsilon} \mathrm{Mo}_{\varepsilon} \mathrm{Si}_{1.75-\delta} \mathrm{Ge}_{\delta}$. The four parameters investigated by the Taguchi method are thus $x, \varepsilon, \delta$ and the processing of the ingot powder, with the values listed in Table IV.

The electrical resistivity, the Seebeck coefficient and the thermal conductivity as a function of temperature were measured for all samples. For all the samples, we calculated $z T$ (see Fig. 4). Here, we only show data taken at $400{ }^{\circ} \mathrm{C}$, highlighting trends between the samples. The corresponding ANOVA table is shown in Table V.

From the four varied parameters, the Mn site fraction, $x$, had the most significant effect on $z T$ for our samples, explaining $70 \%$ of the observed variance, while changing the powder processing condition still accounts for $20 \%$ of the observed variation in $z T$. Due to the complicated structure of HMS, it is difficult to attribute the observed increase of $z T$ with increasing Mn concentration
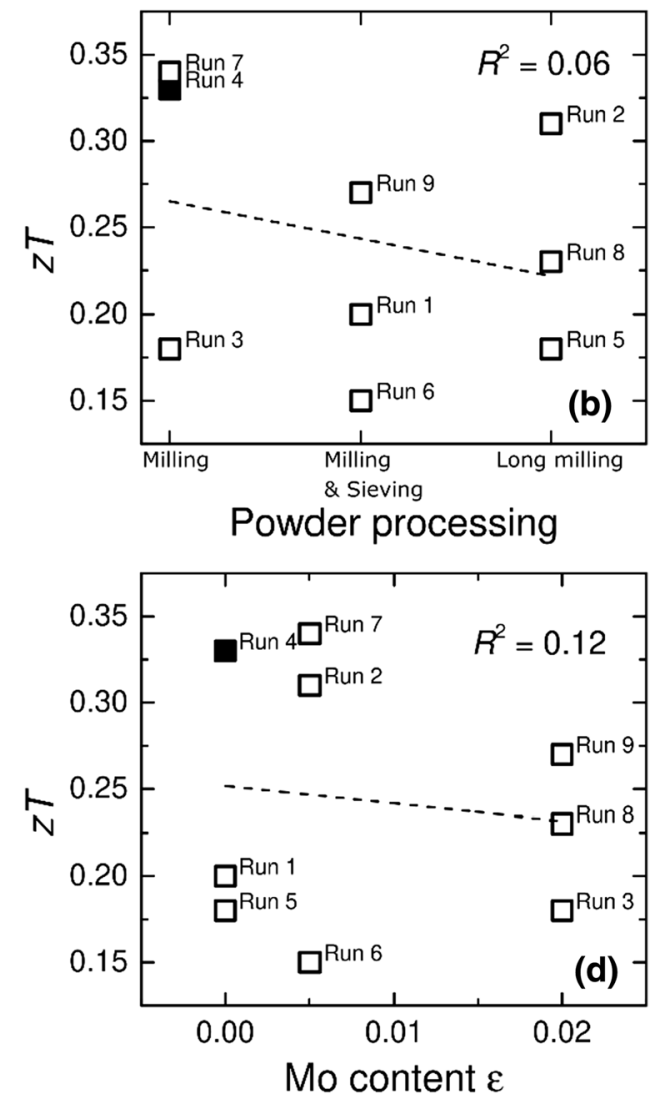

the Mn site. The highlighted sample "Run 4" was used for the module fabrication. 
Table V ANOVA table for data shown in Fig. 4, underlining that the Mn site fraction $x$ has the biggest influence on $z T$

\begin{tabular}{llll}
\hline Parameter & $\begin{array}{l}\text { Degrees of } \\
\text { freedom }\end{array}$ & $\begin{array}{l}\text { Sum of squares } \\
{\left[10^{-3}\right]}\end{array}$ & $\begin{array}{l}\text { Contri- } \\
\text { bution } \\
{[\%]}\end{array}$ \\
\hline Ge content $\delta$ & 2 & 1.9 & 4.6 \\
Powder processing & 2 & 2.6 & 6.4 \\
Mn site fraction $x$ & 2 & 27.5 & 67.3 \\
Mo content $\varepsilon$ & 2 & 8.9 & 21.7 \\
Error & - & N/A & N/A \\
Total & 8 & 40.9 & 100 \\
\hline
\end{tabular}

to a certain microscopic mechanism: It has been argued that slight compositional variations can either change the modulation period (i.e. the $c$ axis length in the commensurate chimney ladder description), ${ }^{37}$ or change the degree of disorder of the sample. ${ }^{38}$ Both mechanisms can have a pronounced effect on the band structure and/or carrier concentration and thus the thermoelectric properties. In addition, there are several other plausible scenarios explaining the influence of Mn concentration on $z T$ : It could influence the average crystallite size of the samples, leading to a variation of scattering processes and thus TE performance. Alternatively, the addition of Mn could lead to the formation of Mn-rich precipitates, not detected by SEM and X-ray diffraction (XRD), which could act as scattering sides or a charge reservoir under a modulation doping hypothesis. More detailed experiments are needed to answer this delicate question, which is beyond the scope of the current paper.

Surprisingly, no clear trend can be detected for the intentional dopants Ge and Mo, which have been shown to have a strong influence of the charge carrier concentration and thus the obtained $z T .{ }^{34-36}$ In order to investigate the absence of a pronounced effect of $\mathrm{Ge}$ and Mo addition on the charge carrier concentration, we analyzed the microstructure by means of SEM. As an example, we show in Fig. 5 the sample of "Run 4," with a nominal composition $\mathrm{Mn}_{1.01} \mathrm{Si}_{1.745} \mathrm{Ge}_{0.005}$. The matrix phase is the targeted HMS, but we also observe minority phases of MnSi and small Ge-rich regions. The presence of $\mathrm{MnSi}$ is very common in HMS samples produced from the melt, due to the higher solidification temperature of MnSi as compared to HMS. ${ }^{39}$ The presence of Ge-rich particles indicates that the added dopant was not resolved (completely) in the matrix structure, increasing the charge carrier concentration and $z T$, but rather precipitated forming an additional Ge-rich phase. We have observed an analogous behavior for the Mo-doped samples. This nonsolubility of the intended dopants is also the reason behind the relatively low $z T$ values for HMS materials, as compared to the best-performing samples in the literature. ${ }^{15,40}$ Melt casting, a scalable and matured technology within the

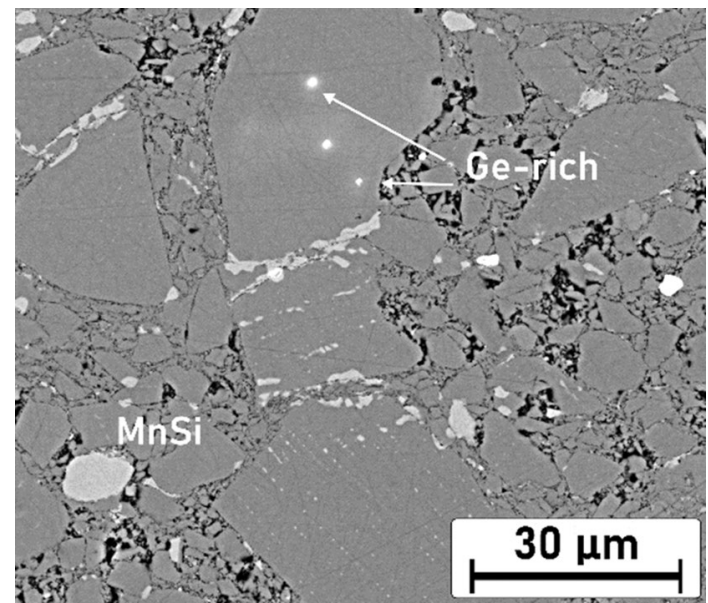

Fig. 5 SEM micrograph of the sample with nominal composition $\mathrm{Mn}_{1.01} \mathrm{Si}_{1.745} \mathrm{Ge}_{0.005}$. The matrix phase is the targeted HMS, but we also observe at least two minority phases, $\mathrm{MnSi}$ and smaller Ge-rich precipitates.

metallurgical industry, may thus be an unsuitable method to synthesize high-performing silicide materials. Different processing steps and methods need to be explored in order to achieve the desired doping effect.

Among the different samples showing similar values of $z T$, we chose the "Run 4" composition, nominally $\mathrm{Mn}_{1.01} \mathrm{Si}_{1.745} \mathrm{Ge}_{0.005}$, to be used for the fabrication of a thermoelectric module. It combines a high $\mathrm{Mn}$ site fraction of 1.01 and the "regular milling" processing parameter, i.e. both parameters, which have been identified as significant and beneficial for a high $z T$ in our ANOVA analysis. The $z T$ value is the second highest among the investigated samples, just insignificantly smaller than that of "Run 7." However, unlike the latter sample, "Run 4" only has a single dopant, $\mathrm{Ge}$, thereby simplifying powder production. The thermoelectric properties of this sample are shown in Fig. 6.

\section{Module Characterization}

Our work aims to investigate the full "value chain" of TE research, spanning from material synthesis using industry-scale methodology, over compositional screening to the fabrication of a TE module. In order to test power generation from the developed materials, we thus fabricated a small module consisting of two $p$ - $n$-pairs, i.e. four legs in total (Fig. 7). As the $n$-type material, we used $\mathrm{Mg}_{2} \mathrm{Si}_{0.3} \mathrm{Sn}_{0.675} \mathrm{Bi}_{0.025}$, with the synthesis route and the thermoelectric properties given elsewhere. ${ }^{41} \mathrm{In}$ an attempt to compensate for the relatively large mismatch in CTE between MGS and HMS, here we used a ductile Pb-based soldering paste, instead of more commonly employed and more high-temperature-resistant thin layer of silver paint. ${ }^{42}$ The metallization scheme employed here must be considered 


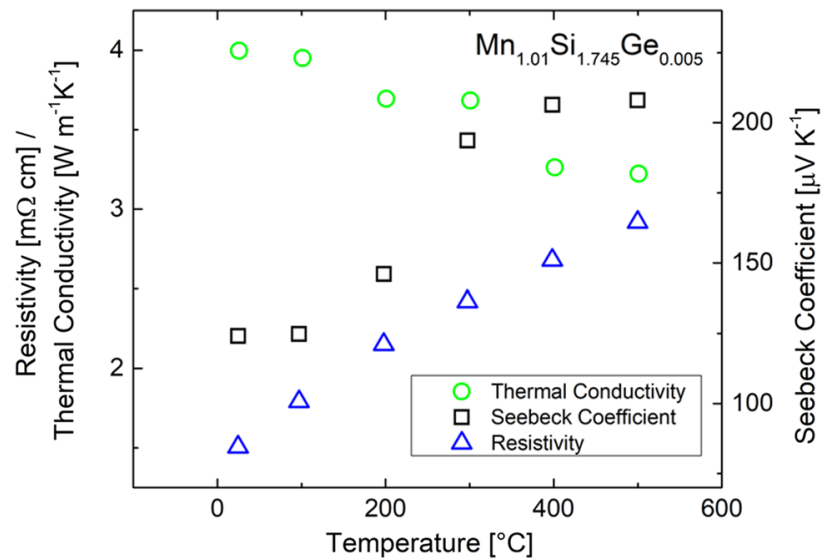

Fig. 6 Thermoelectric properties of the "Run 4" sample, which was used as the $p$-type material of the TE module.

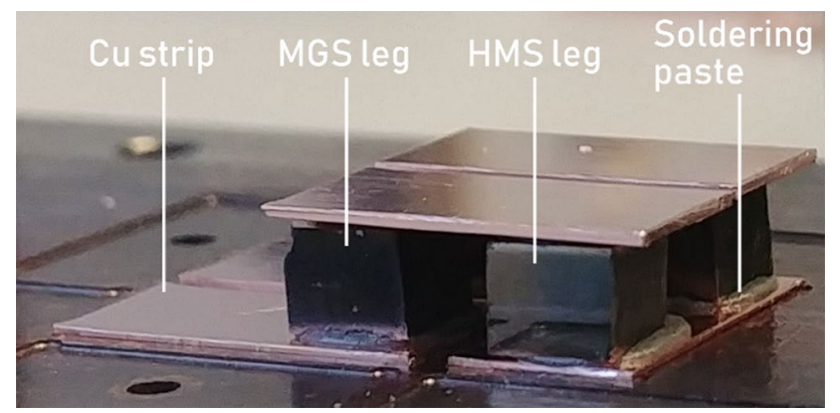

Fig. 7 Photograph of the assembled module.

as an initial, easily implemented method. Thus, we do not expect the resulting module to be particularly robust towards high-temperature exposure, nor stable during long-term operation, and the use of lead raises environmental and legislative concerns. In order to meet these requirements, different bonding materials have to be tested, and additional metallization layers, acting as diffusion barriers, need to be incorporated, which is clearly outside the scope of the current work. Still, we note that our assembly process can be easily scaled up to produce larger devices, independent of the chosen materials. The results of the thermoelectric characterization are summarized in Fig. 8. The slight nonlinearity of the $I-V$ curves, responsible for the skewing of the output power curve, can probably be related to transition of constant $\Delta T$ conditions at high load resistance to constant heat flux $\dot{Q}$ conditions towards higher currents due to the finite duration of the measurement. ${ }^{43}$ The maximum output power for the module was $40 \mathrm{~W}$, with a hot-side temperature of $250{ }^{\circ} \mathrm{C}$ and a temperature difference of $100{ }^{\circ} \mathrm{C}$. In order to compare this output power to reported values from other TE modules, we normalized it with the active area of the TE materials and obtained a power density of $53 \mu \mathrm{W} \mathrm{cm}{ }^{-2}$. This

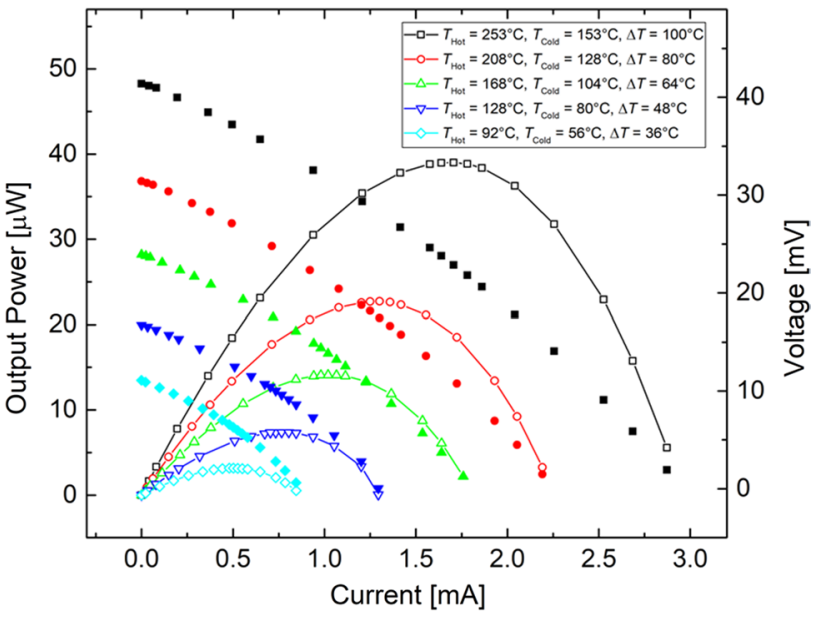

Fig. 8 Output characteristics of the thermoelectric module. The highest output power of $40 \mu \mathrm{W}$ was reached with a hot-side temperature of $250{ }^{\circ} \mathrm{C}$ and a $\Delta T$ of $100{ }^{\circ} \mathrm{C}$.

value is much lower than reported values for silicide-based TE modules in the literature: For example, Funahashi et al. fabricated modules with a power density of $370 \mathrm{~mW} \mathrm{~cm}^{-2},{ }^{20}$ and Skomedal et al. obtained $0.98 \mathrm{~mW} \mathrm{~cm}^{-2},{ }^{18}$ both taken, however, at a higher $\Delta T, 500{ }^{\circ} \mathrm{C}$ and $400{ }^{\circ} \mathrm{C}$, respectively, than in our study. The low generated power output of our model can be related to two main factors: (a) the temperature drop across the electrodes and soldering paste: The theoretically expected open-circuit voltage (OCV) can be calculated from the measured Seebeck coefficient $\alpha_{\mathrm{p}}$ and $\alpha_{\mathrm{n}}$ of the $p$ and $n$-type materials (cf. Fig. 6 and Ref. 41) by Eq. 1

$\mathrm{OCV}_{\text {theoretical }}=n \times\left(\int_{T_{\text {Cold }}}^{T_{\text {Hot }}} \alpha_{\mathrm{p}}(T) d T+\int_{T_{\text {Hot }}}^{T_{\text {Cold }}} \alpha_{\mathrm{n}}(T) d T\right)$

The measured OCV was just $70 \%$ of the theoretically expected value, independent of temperature (Fig. 9), indicating that a significant part of the total temperature drop occurred in regions not contributing to the thermoelectric voltage generation. In other words, the thermal contact between $\mathrm{Cu}$ strips and TE leg materials of our device was poor. (b) The internal resistance of the produced four-leg module was more than $10 \Omega$, which is much larger than the $\approx 10 \mathrm{~m} \Omega$ expected from the ideal intrinsic resistance of the individual legs and neglecting all contributions from electrodes and contact resistance. In order to differentiate between the contact resistance between the solder-Cu and $\mathrm{Cu}-\mathrm{TE}$ material interfaces, we measured the total resistance of metallized and soldered $p$ - and $n$-type legs. The resistance of a leg with a $\mathrm{Cu}$ metallization layer was $50 \mathrm{~m} \Omega$ and $170 \mathrm{~m} \Omega$ for $p$ - and $n$-material, respectively, and increased to $2.0 \Omega$ and $2.3 \Omega$ for the soldered legs. The high total resistance of the module was thus related to the poor contact 


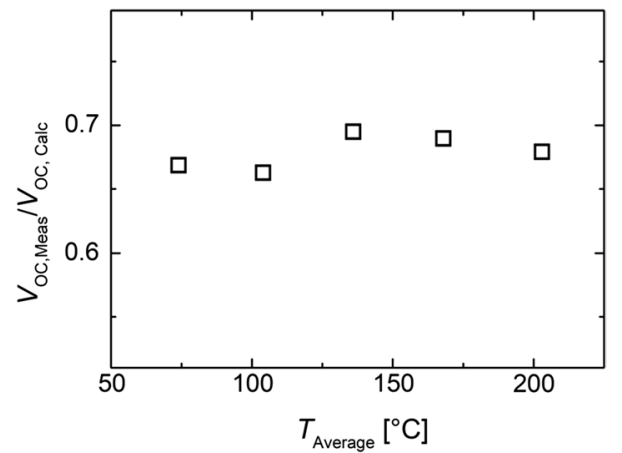

Fig. 9 Ratio of the measured open-circuit voltage to the expected voltage, calculated from the properties of the individual materials. More than $30 \%$ of the total temperature difference is not converted into TE voltage, indicating a poor thermal contact between the contact strips and the TE materials.

quality between the soldering paste and the metallized legs and must be improved in further studies. In a similar study, using different, high-temperature stable contacting methods, an initial heating of the device to $550{ }^{\circ} \mathrm{C}$ was necessary to reduce the internal resistance from several $\mathrm{Ohms}$ to below $100 \mathrm{~m} \Omega .{ }^{18}$ For our module, this was unfortunately unfeasible due to the low melting point of the used $\mathrm{Pb}$-based soldering paste.

\section{Conclusions}

In summary, we have built and characterized a thermoelectric module based on $n$-type $\mathrm{Mg}_{2} \mathrm{Si}$ and $p$-type $\mathrm{Mn}_{1.01} \mathrm{Si}_{1.745} \mathrm{Ge}_{0.005}$ legs. We used a fractional experimental design based on Taguchi methods to find suitable sintering conditions and chemical composition of the $p$-type material. This approach allows the screening of a multidimensional parameter space with a dramatically reduced number of individual samples. The four-leg module was fabricated by a scalable assembly process using a $\mathrm{Pb}$-based soldering paste. The module generated an open-circuit voltage of 35 $\mathrm{mV}$ and a maximum output power density of $53 \mu \mathrm{W} \mathrm{cm}{ }^{-2}$ with a hot-side temperature of $250{ }^{\circ} \mathrm{C}$ and a $\Delta T$ of $100{ }^{\circ} \mathrm{C}$. The low TE output power density was related to the high contact resistance between $\mathrm{Cu}$ contact strips and the TE materials, underlining the importance of improved metallization schemes for TE module assembly, in particular for high-temperature applications.

Acknowledgments The authors gratefully acknowledge funding from the Research Council of Norway (Project No. 269326). J.S.G., O.M.L., and M.S. acknowledge internal financial support from SINTEF to write this publication. J.S.G. is grateful to the technical staff at NTNU for helping with the SPS experiments. J.d.B. and G.C.-H. would like to gratefully acknowledge the endorsement from the DLR Executive Board Member for Space Research and Technology and the financial support from the Young Research Group Leader Program.

Funding Open access funding provided by SINTEF AS.

Conflict of interest The authors declare no conflict of interest.

Open Access This article is licensed under a Creative Commons Attribution 4.0 International License, which permits use, sharing, adaptation, distribution and reproduction in any medium or format, as long as you give appropriate credit to the original author(s) and the source, provide a link to the Creative Commons licence, and indicate if changes were made. The images or other third party material in this article are included in the article's Creative Commons licence, unless indicated otherwise in a credit line to the material. If material is not included in the article's Creative Commons licence and your intended use is not permitted by statutory regulation or exceeds the permitted use, you will need to obtain permission directly from the copyright holder. To view a copy of this licence, visit http://creativecommons.org/licenses/by/4.0/.

\section{References}

1. J.R. Sootsman, D.Y. Chung, and M.G. Kanatzidis, Angew. Chem. Int. Ed. 48, 8616 (2009).

2. G.J. Snyder, and E.S. Toberer, Nat. Mater. 7, 105 (2008).

3. S. Chen, and Z. Ren, Mater. Today 16, 387 (2013).

4. T. Zhu, C. Fu, H. Xie, Y. Liu, and X. Zhao, Adv. Energy. Mater. 5, 1500588 (2015).

5. C. Uher, In Semiconductors and Semimetals, T.M. Tritt, Editor. Elsevier 2001. p. 139-253.

6. S.M. Kauzlarich, S.R. Brown, and G. Jeffrey Snyder, Dalton Trans. 21, 2099 (2007).

7. A. Nozariasbmarz, A. Agarwal, Z.A. Coutant, M.J. Hall, J. Liu, R. Liu, A. Malhotra, P. Norouzzadeh, M.C. Öztürk, V.P. Ramesh, Y. Sargolzaeiaval, F. Suarez, and D. Vashaee, Jpn. J. Appl. Phys. 56, 05DA04 (2017).

8. P. Gao, I. Berkun, R.D. Schmidt, M.F. Luzenski, X. Lu, P. Bordon Sarac, E.D. Case, and T.P. Hogan, J. Electron. Mater. 43, 1790 (2014).

9. D.C. Ramirez, L.R. Macario, X. Cheng, M. Cino, D. Walsh, Y.-C. Tseng, and H. Kleinke, ACS Appl. Energy Mater. 3, 2130 (2020).

10. S.K. Bux, M.T. Yeung, E.S. Toberer, G.J. Snyder, R.B. Kaner, and J.-P. Fleurial, J. Mater. Chem 21, 12259 (2011).

11. K. Mars, H. Ihou-Mouko, G. Pont, J. Tobola, and H. Scherrer, J. Electron. Mater. 38, 1360 (2009).

12. T. Sakamoto, T. Iida, A. Matsumoto, Y. Honda, T. Nemoto, J. Sato, T. Nakajima, H. Taguchi, and Y. Takanashi, J. Electron. Mater. 39, 1708 (2010).

13. H. Kamila, P. Sahu, A. Sankhla, M. Yasseri, H.-N. Pham, T. Dasgupta, E. Mueller, and J. de Boor, J. Mater. Chem. A 7, 1045 (2019).

14. J. de Boor, T. Dasgupta, U. Saparamadu, E. Müller, and Z.F. Ren, Mater. Today Energy 4, 105 (2017).

15. N.L. Okamoto, T. Koyama, K. Kishida, K. Tanaka, and H. Inui, Acta Mater. 57, 5036 (2009).

16. Y. Gelbstein, J. Tunbridge, R. Dixon, M.J. Reece, H. Ning, R. Gilchrist, R. Summers, I. Agote, M.A. Lagos, K. Simpson, C. Rouaud, P. Feulner, S. Rivera, R. Torrecillas, M. Husband, J. Crossley, and I. Robinson, J. Electron. Mater. 43, 1703 (2014). 
17. K.R. Tarantik, J.D. König, M. Jägle, J. Heuer, J. Horzella, A. Mahlke, M. Vergez, and K. Bartholomé, Mater. Today: Proceed. 2, 588 (2015).

18. G. Skomedal, L. Holmgren, H. Middleton, I.S. Eremin, G.N. Isachenko, M. Jaegle, K. Tarantik, N. Vlachos, M. Manoli, T. Kyratsi, D. Berthebaud, N.Y. Dao Truong, and F. Gascoin, Energy Convers. Manage. 110, 13 (2016).

19. I. Aoyama, H. Kaibe, L. Rauscher, T. Kanda, M. Mukoujima, S. Sano, and T. Tsuji, Jpn. J. Appl. Phys. 44, 4275 (2005).

20. R. Funahashi, Y. Matsumura, T. Barbier, T. Takeuchi, R.O. Suzuki, S. Katsuyama, A. Yamamoto, H. Takazawa, and E. Combe, J. Electron. Mater. 44, 2946 (2015).

21. H.S. Kim, K. Kikuchi, T. Itoh, T. Iida, and M. Taya, Mater. Sci. Eng., $B$ 185, 45 (2014).

22. J. Antony, Design of Experiments for Engineers and Scientists (Amsterdam: Elsevier, 2014).

23. T. Kyratsi, and M. Ioannou, J. Electron. Mater. 42, 1604 (2013).

24. R.A. Kishore, P. Kumar, and S. Priya, SUT J. Math. 2, 175 (2018).

25. D. Ji, Z. Wei, S. Mazzoni, M. Mengarelli, S. Rajoo, J. Zhao, J. Pou, and A. Romagnoli, Energy Convers. Manage. 172, 507 (2018).

26. H. Terzioğlu, Measurement 149, 106992 (2020).

27. R.A. Kishore, M. Sanghadasa, and S. Priya, Sci. Rep. 7, 16746 (2017).

28. W.-H. Chen, S.-R. Huang, and Y.-L. Lin, Appl. Energy 158, 44 (2015).

29. S. Ahmad, A. Singh, R. Basu, S. Vitta, K.P. Muthe, S.C. Gadkari, and S.K. Gupta, J. Electron. Mater. 48, 649 (2019).

30. A. Kanatzia, C. Papageorgiou, C. Lioutas, and T. Kyratsi, J. Electron. Mater. 42, 1652 (2013).

31. M. Schrade, H. Fjeld, T. Norby, and T.G. Finstad, Rev. Sci. Instrum. 85, 103906 (2014).
32. R. Schuler, R. K. Madathil, and T. Norby, Rev. Sci. Instrum. 92, 043902 (2021). https://doi.org/10.1063/5.0032698.

33. D.Y.N. Truong, H. Kleinke, and F. Gascoin, Dalton Trans. 43, 15092 (2014)

34. D.Y. Nhi Truong, D. Berthebaud, F. Gascoin, and H. Kleinke, $J$. Electron. Mater. 44, 3603 (2015).

35. A.J. Zhou, T.J. Zhu, X.B. Zhao, S.H. Yang, T. Dasgupta, C. Stiewe, R. Hassdorf, and E. Mueller, J. Electron. Mater. 39, 2002 (2010).

36. P.Y. Pichon, P. Berneron, J. Levinsky, A. Burema, G. Blake, D. Berthebaud, S. Gascoin, F. Gascoin, S. Hebert, J. Amtsfeld, T. Hommels, M. Huijben, J. de Boor, E. Müller, C. Navone, and A. Schönecker, J. Alloys Compd. 832, 154602 (2020).

37. Y. Miyazaki, D. Igarashi, K. Hayashi, T. Kajitani, and K. Yubuta, Phys. Rev. B 78, 214104 (2008).

38. L. Akselrud, R. Cardoso Gil, M. Wagner-Reetz, and Y. Grin, Acta Crystallogr. B 71, 707 (2015).

39. T.D. Johannes de Boor, Eckhard Mueller, Materials Aspect of Thermoelectricity. ed. C. Uher (Boca Raton: CRC Press, 2016), pp. $159-218$.

40. W.-D. Liu, Z.-G. Chen, and J. Zou, Adv. Energy. Mater. 8, 1800056 (2018).

41. N. Farahi, C. Stiewe, D.Y.N. Truong, J. de Boor, and E. Müller, RSC Adv. 9, 23021 (2019).

42. A.d.P. Shyikira, G. Skomedal, and P. Hugh Middleton Materials Today: Proceedings https://doi.org/10.1016/j.matpr.2020.05.193 (2020)

43. G. Min, Meas. Sci. Technol. 25, 085009 (2014).

Publisher's Note Springer Nature remains neutral with regard to jurisdictional claims in published maps and institutional affiliations. 